\title{
Design and Prospect of Several Kinds of Round Bamboo Furniture Fittings that Can Be Standardized
}

\author{
Wenying Dong \\ College of Forest \\ Sichuan Agricultural University \\ Chengdu, China 611130 \\ Central South University of Forestry and Technology \\ Changsha, China 410000 \\ Xingyin Liu \\ College of Forest \\ Sichuan Agricultural University \\ Chengdu, China 611130
}

\author{
Yi'en Geng \\ College of Forest \\ Sichuan Agricultural University \\ Chengdu, China 611130
}

\begin{abstract}
As a sustainable development material, bamboo has become a hot research object in recent years with its characteristics of environmental friendly. By analyzing the reasons for the market situation of round bamboo furniture, the plastic, metal and rubber materials are used in the design of the round bamboo furniture fittings production, in order to achieve the round bamboo furniture with solid structure, removable parts and standardizing fittings. To a certain extent, standardized production has improved the design-manufacture present situation and promoted the market value of round bamboo furniture.
\end{abstract}

Keywords-round bamboo furniture; fittings; standardized production

\section{INTRODUCTION}

In China, the round bamboo furniture is usually produced by handcraft. It has a fixed production method, tedious production process, backward technology and old furniture, so the furniture style is out of date and production standards are not unified, resulting in low production efficiency and not to be loved by consumers. In the furniture fittings, round bamboo furniture still adopts the traditional fixed structure, and it's no removable brings great difficulties to the replacement and maintenance of parts. These structures problems affect the development of the production, technology, modeling and function of the round bamboo furniture, which has led to the round bamboo furniture industry not been greatly developed. It is far behind the furniture enterprises using other materials in the aspect of scale and mass production.

\author{
Yu Zhao \\ College of Forest \\ Sichuan Agricultural University \\ Chengdu, China 611130
}

\section{INVESTIGATION AND ANALYSIS OF THE MARKET SITUATION OF ROUND BAMBOO FURNITURE}

\section{A. Market Status of Round Bamboo Furniture}

In 1995 the third national industrial census data show that: the furniture enterprise in the villages and towns above, the wooden furniture manufacturing companies share reached $79 \%$, while only $3 \%$ bamboo rattan furniture manufacturing enterprises. At present, the number of enterprises specializing in producing round bamboo furniture is small and the scale is small. In addition, there are some bamboo integrated part of the factory workshop production round bamboo furniture, regardless of size, but the production unit is basically the manual production, the main production equipment to dismiss knife, knife, knife, scraper, gang saw, planer, chisels, hammers, ruler, such as caliper, hand electric drill tool and the working bench, burner, baking column, transverse saw, simple equipment such as boiling pool [1], so the mechanization degree is low.

In recent years, although the productivity of round bamboo furniture in our country has a certain improvement and the enhancement compared with the traditional round bamboo furniture, but far less than other furniture such as wooden furniture, bamboo integrated furniture, especially in the case of large-scale mass, standardized [2]. In addition, our country round bamboo furniture product is in inferior position in furniture market due to the problem of old style and old specification and complicated manufacture.

\section{B. Key Factors of the Slow Development of Round Bamboo Furniture}

1) Furniture materials and structures: There are four regrettable defects in bamboo due to chemical composition and tissue structure. Bamboo is more susceptible to inset decay, corrosion, moldy, burning and uneven color than 
wood, greatly limiting the development and utilization of bamboo timber [3]. In addition, the traditional bamboo furniture is connected to the fixed structure which cannot be dismantled. This fixed structure hinders the improvement of production efficiency, increases the cost and difficulty of the transportation, packaging and storage of the products, and is also not conducive to the advantages of recycling and reuse of bamboo [4]. Technology used more punch, bending, etc., the phenomena of dry shrinkage and wet expansion often appear in the use of bamboo without change of the physical and chemical properties, which causes, the phenomenon of the cracking deformation, influence of furniture not only beautiful, also greatly shorten the service life. All these round bamboo furniture structural problems, will affect the round bamboo furniture production, the development of technology, modelling, function, leading to a round bamboo furniture industry in the moment for a long time did not get great development and progress. Therefore, the development of round bamboo furniture industry must first overcome the "structural problem" of round bamboo furniture [2].

2) Production process and equipment: The main process flow of round bamboo furniture production is: material selection, pharmaceutical treatment, drying, blanking, roasting bamboo, car bamboos, marking, inking, gelding bamboo, tenon board, assembly, surface painting, etc. Some small enterprises do not carry out the material treated with medicament, no dry equipment, the lack of standard technology. It makes the product easily produce moth damage, mildew, cracking, deformation, shedding and so on, which seriously affecting the safety, service life, function and aesthetics of furniture [1]. In this series of processes, the connection of the main material is also a complicated and extremely important process. Round basic connection with inclusion of bamboo furniture, scarf, and receive, log core, butt, tee, cross, one word, and wind, etc. These connection methods cannot be used alone, and often need several ways to match them. However, the existing connector cannot be applied to most bamboo, such as the built-in connector, metal connector and socket connector in the production process, the diameter and thickness of the main material should be taken into account.

3) Design level and aesthetic value: With the rapid development of today's era, the city is changing with each passing day. Stone and wood are replaced by reinforced concrete, and the cultural ideas from all over the world have been imported into China, which has caused a shock to our traditional culture. In the selection of furniture materials, materials such as metal and cloth art which have a strong modern style can attract the eyes of the young people more, and the leather, wood are more in line with the aesthetic of the middle-aged people. Compared with the furniture influenced by foreign culture, the traditional round bamboo furniture seems to be single in shape and old in color, which is not conform to the fashion cycle. the style and design of round bamboo furniture mostly follow the old system, little innovation, slow update speed, which make the bamboo furniture do not meet the modern aesthetic requirements. At the same time, the aesthetic standards of contemporary society and consumers are gradually improving. The traditional appearance of round bamboo furniture is in contradiction with the contemporary aesthetic form. The main reason for the difficult development of round bamboo furniture is that manual production is too labor intensive. And the round bamboo furniture still adopts the traditional fixed structure, which ca not be disassembled. This brings great difficulties to the replacement and maintenance of the parts. Therefore, the maintenance of the round bamboo furniture can only be partially repaired on the basis of the original furniture, but not the replacement of the parts [5]. Bamboo can't mass production, the market also cannot mass production of bamboo furniture fittings, round bamboo furniture production cannot mechanization production, the production efficiency are improved, caused the round bamboo furniture is now in the awkward position of furniture market, it is difficult to competition among the furniture industry.

\section{DESIGN IDEAS OF ROUND BAMBOO FURNITURE FITTINGS}

Based on the above summary and analysis of key factors, starting with the round bamboo furniture connection mode and combining with Sichuan commonly used bamboo diameter range, design a few easy processing and standardization production round bamboo furniture fittings. The purpose is to improve the structural stability and durability of the existing round bamboo furniture, and to try to disassemble the round bamboo furniture, improve the production efficiency, and improve the market value of round bamboo furniture.

Circle diameter and wall thickness of bamboo is a problem that must be considered in the design of fittings, by fitting the characteristics of the materials increase the elastic fittings, solve the two problems one or two, then round bamboo furniture fittings of mass production can be achieved.

\section{MANUfaCture TeChNOLOGY OF ROUND BAMBOO FURNITURE FITTINGS}

The production and application of connecting parts will bring great convenience to the production of round bamboo furniture. The traditional production technology of round bamboo furniture not only damages round bamboo to the connection of main material, but also reduces the service life of the round bamboo furniture. And mass production can be fitting saves time and labor, also won't damage to round bamboo, so that the round bamboo furniture more modern production technology, also conforms to the current development trend. It is round a progress of production technology of bamboo furniture. 


\section{A. Processing Technology of Plastic Parts}

Traditional terraced and wrapped socket connectors in line-styled in "Fig. 1" are unable to adapt to a variety of the diameter of the round bamboo and easy to be installed or loosening problem in connection with the round bamboo. It need to special socket connector after measure the round bamboo diameter, which is very inconvenient in the production process. And the nylon sawtooth belt fasteners are made of nylon, which makes fully use the advantages of nylon, such as non-toxic, tasteless, mildew-proof and good mechanical properties such as tensile compressive and abrasion resistance. It can control the loose of pipe fitting mouth through serrated band to meet the purpose of various diameter round bamboo, as well as it can also be mass produced to save labor and production time. This type of connection only contacts with the outer wall of the round bamboo, we can ignore the problems caused by the thickness of the round bamboo wall.
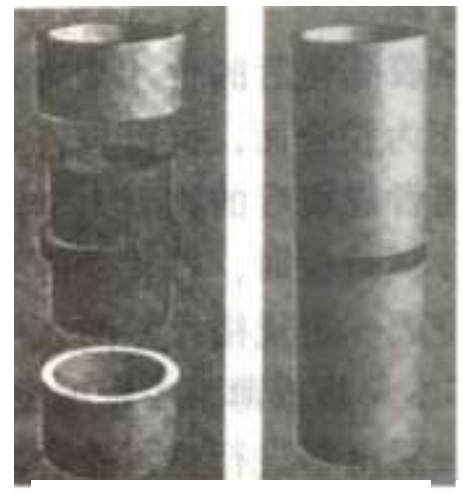

Stepped socket connector in line-styled
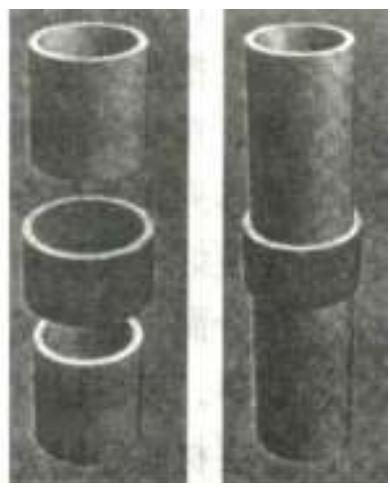

Package socket connector in line-styled
Fig. 1. Traditional socket connector in line-styled.

The joint is simple in structure and consists of inner and outer layers of pipe material and serrated band at pipe opening. The inner pipe has three notches at each end, in order to accommodate round bamboo with a larger diameter than the fitting. The serrated band is serrated on the outside of the end, and three clasps are arranged on the inside of the other end. The internal design of the clasp is an inclined plane adapted to the serrated Angle in "Fig. 2" and "Fig. 3". The serrated band is designed to fit round bamboo with inserts the clasp to tighten and draws the clasp to relax.

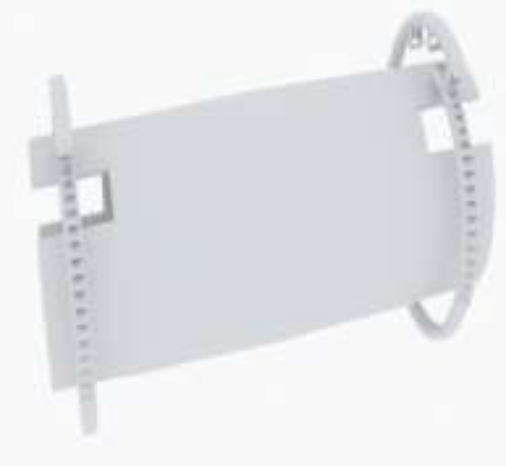

Fig. 2. Nylon serrated band in line-styled.

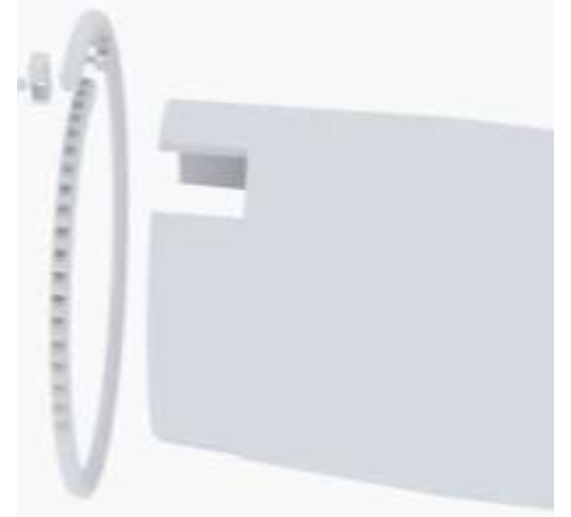

Fig. 3. Explosion diagram of nylon serrated band connector in line-styled.

\section{B. Processing Technology of Rubber and Metal Parts}

Traditional T-type socket joints in "Fig. 4" also cannot solve the problems caused by the diameter of round bamboo. This connector "Fig. 5" with synthetic rubber, aluminum and stainless steel as the material. Synthetic rubber with natural rubber excellent abrasion resistance, heat-resistant, ageing resistance, corrosion resistance or oil resistant performance, make it high elasticity under the condition of use which can adapted to round bamboo diameter from two aspects of inside and outside, in order to adapt to a variety of diameter round bamboo purposes This type connector is in contact with both the inner and outer walls of the round bamboo when using it. We can adapt the diameter and wall thickness of round bamboo by using the elasticity of high elastic rubber inside and outside. Stainless steel is resistant to corrosion of weak corrosive medium such as air, steam and water, and chemical corrosive medium such as acid, alkali and salt; Aluminum has the characteristics of light weight and corrosion resistance; these two metal materials have superior performance. This connection uses the method of combining the inner sleeve with the external connection, and solves the problems caused by the diameter and wall thickness of round bamboo.
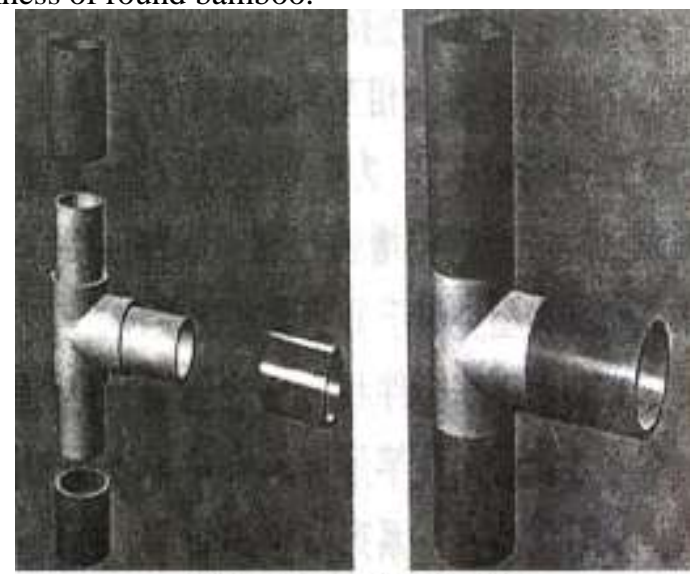

T-type socket connector and assembly diagram

Fig. 4. Traditional T-type socket connector. 


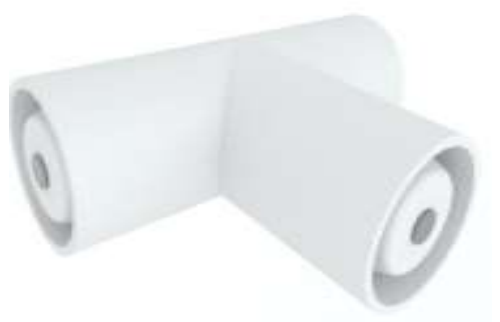

Fig. 5. Rubber and stainless-steel $\mathrm{T}-$ type connector.

The fittings for inside and outside two layer structure. The inner layer in stainless steel metal pipe as the main body, port installation frustum of a cone shape rubber, fix rubber and stainless steel with aluminum sheet to prevent the frustum of a cone rubber drop from on the stainless steel pipe fittings; The outer layer is a thin layer of tubular rubber, and the middle part of the thin layer is connected with stainless steel pipe, so that the inner and outer layers are fixed together in "Fig. 6". The function of rubber thin layer: (1) To make the connection between round bamboo and fittings more stable; (2) Block the inner structure and the joint formed by connecting the round bamboo to the joint.

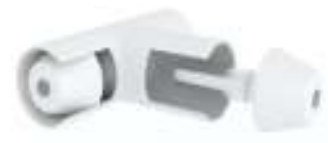

Fig. 6. Explosion diagram of rubber and connector.
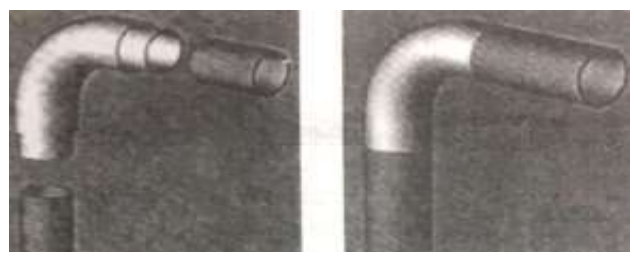

L-type stepped socket connector and assembly diagram

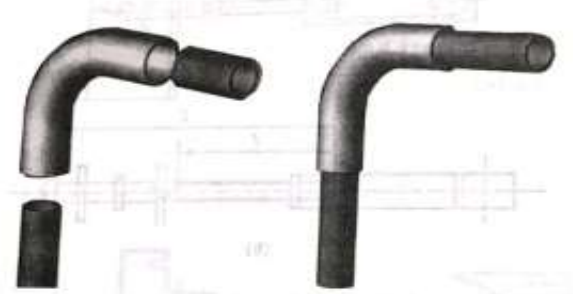

L-type package socket connector and assembly diagram

\section{Processing Technology of Rubber and Plastic Parts}

Traditional L-type connectors are generally divided into two types: terraced socket and inner socket "Fig. 7". They are basically the same as other conventional connectors of the same type, both of which are fixed and single in material modeling function and other aspects, which greatly restrict their use. The rubber and plastic L-type connector "Fig. 8" mainly adopts inner connection and elastic material to adapt to the diameter of round bamboo, with synthetic rubber and low-pressure polyethylene plastic as material. Polyethylene has high water resistance and can keep its performance unchanged in long-term contact with water. The advantages of low pressure polyethylene, such as wear-resistant, corrosion-resistant, heat resistant and good insulation, and to tighten the rotating nut to adapt to a variety of diameter round bamboo purposes. This kind of connector only contacts with the outer wall of round bamboo, and problems caused by the thickness of round bamboo wall can be ignored when using it as in "Fig. 9".

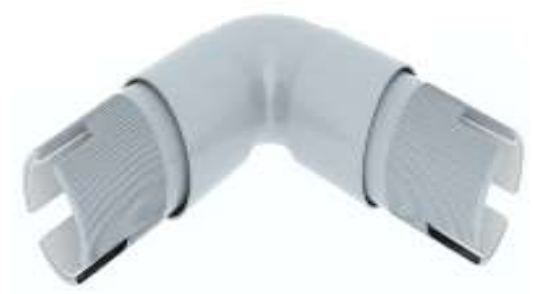

Fig. 8. Rubber, plastic L-type connector.

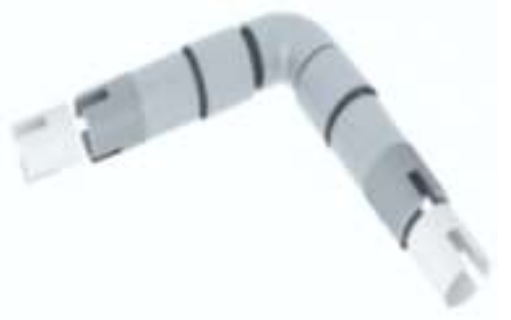

Fig. 9. Explosion diagram of rubber and plastic L-type connector.

\section{CONCLUSION}

\section{A. It Is of Great Significance to the Progress of Production} Technology of Round Bamboo Furniture

Sustainable development strategy is a strategy that must be implemented in China's modernization construction. It is crucial to pay attention to the conservation and maximum utilization of resources. And standardization of fittings saves time and labor in the process of production, to maximize the advantage of the performance of the round bamboo, drive round bamboo furniture production technology innovation, so that green, ecological and sustainable development of bamboo furniture blossom new brilliance [6], This is of great significance to the development and use of round bamboo furniture.

Fig. 7. Traditional L-type socket stainless-steel T-type connector. 


\section{B. The Inevitable Direction of the Development of Round Bamboo Furniture Industry}

At present most of the round bamboo furniture structure is not the $\mathrm{KD}$ furniture. Under the impact of the rapid development of the new e-commerce network, the traditional furniture enterprises have been transformed into network sales. Logistics transportation requires that the furniture structure can realize can be disassembled or folded, which is beneficial to the packaging storage and transportation of products, so as to reduce the cost of enterprise [2]. Not only that, the industrialization and mechanization of the rapid development today, the large-scale, standardized quantification of products has also become a test of the furniture industry. In terms of the production of the round bamboo furniture, fittings is an important component of the standardized determines whether it round bamboo furniture product quality, whether it can be mass determines the size of the round bamboo furniture production and efficiency. The orientation of these market changes leads to the mass production of round bamboo furniture, so the standardized production of its fittings is particularly important.

On the basis of these conditions, the development of round bamboo furniture has become an indispensable link in the future, and the improvement of production efficiency of round bamboo furniture has become the primary problem for the development of round bamboo furniture. The application of standardized fittings is the way to improve the production efficiency of round bamboo furniture. Therefore, the round bamboo furniture fittings that can be standardized have an infinite future.

\section{ACKNOWLEDGMENT}

This work was financially supported by the major project of humanities and social sciences of education department of Sichuan province (16SA0006), the "Key Laboratory of Wood Industry and Universities", the project of Undergraduate Scientific Research Interest of Sichuan Agricultural University(2018141).

\section{REFERENCES}

[1] Wu Zhihui, Li Jiqing, Yuan Zhe. Bamboo rattan furniture manufacturing technology [M]. Beijing: China forestry press, 2009: 6 .

[2] He Euilin. Research on the design of round bamboo furniture based on structural innovation [D]. Central South University of Forestry and Technology, 2016.

[3] Hui Chaomao, Yang Yuming. Chinese bamboo cultivation and utilization handbook [M]. Beijing: China forestry press, 2002: 159.

[4] Zhang Chen. Feasibility study on the design of round bamboo furniture based on mass production [D]. Donghua university, 2006.

[5] Yang Lingyun. Research on the design of new joint structure for round bamboo furniture [J]. Bamboo research journal, 2015, 34(01): 26-30.

[6] He Ruilin, Zhou Ziyun, Zhang Zhongfeng. Innovative design of round bamboo furniture structure [J]. Forest products industry, 2016, $43(04)$. 